\title{
EFEITO DOS HERBICIDAS DICLOSULAM E METOLACHLOR NO CONTROLE EM PRÉ-EMERGÊNCIA DE Sida rhombifolia NA CULTURA DA SOJA
}

\author{
Dionisio L. P. Gazziero ${ }^{1}$, Elemar Voll ${ }^{1}$, Cléber Daniel de G. Maciel², \\ José Roberto Scaramuzza-Jr. ${ }^{3}$ e Cássio E. C. Prete ${ }^{4}$
}

\footnotetext{
${ }^{1}$ Embrapa Soja, Caixa Postal 231, Londrina, PR

2 Universidade Estadual de Maringá. Maringá, PR

${ }^{3}$ Faculdade de Agronomia de Garça. Garça, SP

${ }^{4}$ Universidade Estadual de Londrina, PR
}

86001-970. E-mail: gazziero@cnpso.embrapa.br

RESUMO

Com o objetivo de avaliar os efeitos dos herbicidas diclosulam e metolachlor, aplicados sozinhos ou em mistura, no controle em pré-emergência de $S$. rhombifolia na cultura da soja, foi instalado um experimento em Latossolo Roxo distrófico, em Londrina-PR, no ano agrícola 1996/97.O delineamento experimental foi o de blocos casualizados com quatro repetições. Os tratamentos foram: diclosulam a 25 e $35 \mathrm{~g} / \mathrm{ha}$, diclosulam + metolachlor a 25+1200, 25+1800, 35+1200 e 35+1800 g/ha e metolachlor a 1200,1800 e $2400 \mathrm{~g} / \mathrm{ha}$. Como padrão foram utilizados chlorimuron + lactofen $(12,5+96 \mathrm{~g} / \mathrm{ha})$ e testemunhas, com capina e sem capina. A cultivar BR-16 foi semeada em 05/12/96 e a aplicação foi realizada na mesma data, utilizando-se pulverizador costal pressurizado $\mathrm{CO}_{2}$, equipado com barra de quatro bicos $110-02 \mathrm{XP}$, pressão de $2,45 \mathrm{~kg} / \mathrm{cm}^{2}$ e volume de pulverização de $2001 /$ ha. Diclosulam + metolachlor e diclosulam sozinho, ambos na menor dose, controlaram satisfatoriamente a guanxuma, não diferindo estatisticamente dos tratamentos com as doses maiores. Metolachlor sozinho, nas maiores doses, apresentou nível de controle em torno de $80 \%$, diferindo estatisticamente dos melhores tratamentos. Devido às condições climáticas adversas, o padrão chlorimuron + lactofen foi aplicado quando as plantas de guanxuma estavam com 4 a 12 folhas e 5 a $25 \mathrm{~cm}$ de altura. Aos 49 dias após aplicação verificou-se $80 \%$ de controle, equivalendo ao tratamento com metolachlor a $2400 \mathrm{~g} / \mathrm{ha}$. A fitotoxicidade do diclosulam sozinho e em mistura com o metolachlor atingiu níveis de controle inferiores a 5\%. Conclui-se que o diclosulam (25 $\mathrm{g} / \mathrm{ha})$ e diclosulam + metolachlor $(25+1200 \mathrm{~g} / \mathrm{ha})$, controlaram eficientemente a guanxuma.

Palavras-chave: controle químico, guanxuma, planta daninha, Glycine max.

\section{ABSTRACT \\ Effect of preemengence herbicides diclosulam and metolochlor for Sida rhombifolia control in soybean}

An experiment was conducted on a Cerrado oxisol during 1996/97. The objective of experiment was to evaluate the effects of diclosulam and metolochlor alone or in combination, in preemergence control of Sida rhombifolia in soybean. The experimental design was a complete randomized block with four replications. Treatments were as follows: diclosulam at 25 and $35 \mathrm{~g} / \mathrm{ha}$, diclosulam + metolachlor at 25+1200, 25+1800, 35+1200 and 35+1800 g/ha, metolachlor at 1200,1800 and $2400 \mathrm{~g} / \mathrm{ha}$, and a tank mix of chlorimuron + lactofem at 1.5+96 g/ha used for comparison. A weedy and a weeded control were also included. Soybean seeds (BR-16 cultivar) were sowed December 5. 1996 and herbicides applied in the same day using a back sprayer, with four 110-02XP nozzles, operated at 2,45 kg/ $\mathrm{cm}^{2}$ to deliver 200 1/ha. Diclosulam and diclosulam + metolachlor at the smaller rates gave good control of $S$. rhombifolia, with no significant differences to the highest dosages. Metolachlor at 1800 and $2400 \mathrm{~g} /$ ha controlled about $80 \%$ of the weed. These results were significantly different from the results with diclosulam and diclosulam + metolochlor. Due to adverse climate condition in the area, chlorimuron + lactofen was 
applied when S. rhombifolia had 4-12 leaves and 5-25 cm high. After 49 days chlorimuron + lactofen had controlled $80 \%$ of $S$. rhombifolia, wich was equal to metolachlor $(2400 \mathrm{~g} / \mathrm{ha}$ ) results. Diclosulam and diclosulam + metolachlor gave less than $5 \%$ toxicity to soybean. It was concluded that diclosulam at $25 \mathrm{~g} /$ ha and diclosulam + metolachlor at $25+1200 \mathrm{~g} /$ ha gave good control of S. rhombifolia.

Key words: chemical control, weed, Glycine max.

\section{INTRODUÇÃO}

A cultura da soja é uma das principais atividades agrícolas de verão, chegando a atingir em 1996/97 uma área de plantio em torno de 11,3 milhões de hectares. Um dos maiores problemas da cultura é a interferência das plantas daninhas, pois a competição pode ocasionar pressões de ordem direta e indireta que diminuem a qualidade e quantidade do produto final. É necessário que se encontrem alternativas eficientes de controle, que proporcionem aumento no rendimento com mínimo impacto ambiental possível. O meio mais eficaz para se combater as plantas daninhas é o uso combinado de diferentes práticas visando aproveitar os recursos disponíveis, com menor custo e máxima segurança (Deuber, 1992).

A Sida rhombifolia (guanxuma), é considerada uma das principais plantas daninhas da cultura da soja. É uma malvaceae, anual ou perene, subarbustiva, com reprodução por sementes, freqüente em solos cultivados ou não e distribuída geograficamente de norte a sul no Brasil. É uma infestante muito competitiva com as culturas agrícolas devido ao seu profundo sistema radicular (Lorenzi, 1982).

O presente trabalho teve como objetivo avaliar os efeitos causados pelos herbicidas diclosulam e metolachlor, quando aplicados sozinhos ou em mistura, no controle em préemergência da guanxuma na cultura da soja.

\section{MATERIAL E MÉTODOS}

O experimento foi realizado em área de produção comercial da Fazenda São Gregório, em Londrina-PR, no ano agrícola 1996/97. A cultivar BR-16 foi semeada em 05/12/ 96, utilizando-se 20 sementes/metro linear e espaçamento de $0,45 \mathrm{~m}$ entre linhas, em um Latossolo Roxo eutrófico, com $62,6 \%$ de argila, $18,4 \%$ de silte, $18,9 \%$ de areia e $2,2 \%$ de matéria orgânica. O preparo do solo foi feito de forma convencional e os tratos culturais segundo as recomendações técnicas da Embrapa Soja (1996).

A aplicação dos tratamentos com os herbicidas de préemergência diclosulam e metolachlor foi realizada logo após a semeadura. Utilizou-se um pulverizador costal pressurizado a $\mathrm{CO}_{2}$, pressão constante de $2,4 \mathrm{~kg} / \mathrm{cm}^{2}$ barra de 4 bicos tipo leque 110-XP2, espaçados de $0,50 \mathrm{~m}$, proporcionando um volume de pulverização de 200 1/ha. No momento da aplica- ção o solo estava úmido, com temperatura de $29,9^{\circ} \mathrm{C}$ a $5 \mathrm{~cm}$ de profundidade, temperatura ambiente de $31,6^{\circ} \mathrm{C}$, umidade relativa do ar de $85 \%$ e ventos com velocidade de 0 a $4 \mathrm{~km} /$ h. No término da aplicação as condições do ambiente estavam praticamente inalteradas. $O$ tratamento padrão consistiu da mistura dos herbicidas chlorimuron + lactofen, em pós-emergência, realizada 32 dias após a semeadura, quando a soja estava com 3 a 4 folhas trifolioladas e as plantas de guanxuma com 10 a 15 folhas e aproximadamente $25 \mathrm{~cm}$ de altura. Utilizou-se o mesmo equipamento das aplicações anteriores. A temperatura do ar era de $30,0^{\circ} \mathrm{C}$, a do solo $35,3^{\circ} \mathrm{C}$ e a umidade relativa do ar variou de 60 a $70 \%$. O céu se apresentava parcialmente nublado com ventos de 4 a $8 \mathrm{~km} / \mathrm{h}$ e o solo estava seco. $\mathrm{O}$ atraso na pulverização do herbicida em pós-emergência foi devido às condições climáticas adversas, que não permitiram aplicação no estádio de 3 a 4 folhas da planta invasora, conforme previsto.

O número médio de plantas de guanxuma presentes na testemunha sem capina totalizou 69 plantas $/ \mathrm{m}^{2}$. As gramíneas foram controladas com a aplicação, em pós-emergência, do herbicida clethodim (Select) na dose comercial de 0,5 tha.

O experimento foi disposto em parcelas com delineamento em blocos casualizados. As parcelas mediram $2 \mathrm{~m} \times 8$ $\mathrm{m}\left(16 \mathrm{~m}^{2}\right)$, sendo utilizados 12 tratamentos com 4 repetições, incluindo as duas testemunhas, uma capinada e outra sem capina. Na Tabela 1 encontram-se discriminados os tratamentos utilizados com o nome comum e comercial dos produtos, o modo de aplicação, o tipo de formulação, a concentração e as doses utilizadas. Na Tabela 2 encontram-se o nome comum, o nome químico, o grupo químico e a classe toxicológica dos produtos, e na Tabela 3 as precipitações observadas antes e após a instalação do experimento.

As avaliações de controle e fitotoxicidade foram realizadas aos 35, 60 e 91 dias após aplicação (DAA) para os tratamentos em pré-emergência e aos 10,29 e 49 DAA para o tratamento padrão, empregando-se o método de avaliação visual onde 0 representa nenhum controle ou nenhuma fitotoxicidade e 100 representa controle total ou morte das plantas. Também foi dado um conceito sobre o controle em cada parcela (Sociedade Brasileira da Ciência das Plantas Daninhas, 1995).

Os dados obtidos foram submetidos à análise de variância e as médias foram comparadas pelo teste de Tukey a $5 \%$ de probabilidade. 
Tabela 1. Tratamentos, formulação e doses de produtos utilizados em pré-emergência no controle de Sida rhombifolia. Embrapa Soja. Londrina-PR, 1977.

\begin{tabular}{|c|c|c|c|c|c|}
\hline \multicolumn{2}{|c|}{ Tratamento } & \multirow{2}{*}{ Formulação } & \multirow{2}{*}{ Concentração } & \multicolumn{2}{|c|}{ Dose $^{3}$} \\
\hline Nome comum & Nome comercial & & & (g.i.a./ha) & p.c./ha \\
\hline Diclosulam & Spider & G.D.' & $840 \mathrm{~g} / \mathrm{kg}$ & 25 & $30 \mathrm{~g}$ \\
\hline Diclosulam & Spider & G.D. & $840 \mathrm{~g} / \mathrm{kg}$ & 35 & $42 \mathrm{~g}$ \\
\hline Diclosulam + metolachlor & Spider + Dual & G.D./C.E. & $840 \mathrm{~g} / \mathrm{kg}+960 \mathrm{~g} / \mathrm{kg}$ & $25+1200$ & $30 \mathrm{~g}+1250 \mathrm{ml}$ \\
\hline Diclosulam + metolachlor & Spider + Dual & G.D./C.E. & $840 \mathrm{~g} / \mathrm{kg}+960 \mathrm{~g} / \mathrm{kg}-\mathrm{L}$ & $25+1800$ & $30 \mathrm{~g}+1875 \mathrm{ml}$ \\
\hline Diclosulam + metolachlor & Spider + Dual & G.D./C.E. & $840 \mathrm{~g} / \mathrm{kg}+960 \mathrm{~g} / \mathrm{kg}$ & $35+1200$ & $42 \mathrm{~g}+1250 \mathrm{ml}$ \\
\hline Diclosulam + metolachlor & Spider + Dual & G.D./C.E. & $840 \mathrm{~g} / \mathrm{kg}+960 \mathrm{~g} / \mathrm{l}$ & $35+1800$ & $42 \mathrm{~g}+1875 \mathrm{ml}$ \\
\hline Metolachlor & Dual & C.E. ${ }^{2}$ & $960 \mathrm{~g} / 1$ & 1200 & $1250 \mathrm{ml}$ \\
\hline Metolachlor & Dual & C.E. & $960 \mathrm{~g} / 1$ & 1800 & $1875 \mathrm{ml}$ \\
\hline Metolachlor & Dual & C.E. & $960 \mathrm{~g} / 1$ & 2400 & $2500 \mathrm{ml}$ \\
\hline Chlorimuron-ethyl +lactofen & Classic + Cobra & G.D. + C.E. & $250 \mathrm{~g} / \mathrm{kg}+240 \mathrm{~g} / \mathrm{l}$ & $12,5+96$ & $50 \mathrm{~g}+400 \mathrm{ml}$ \\
\hline Testemunha com capina & - & - & - & - & - \\
\hline Testemunha sem capina & - & - & - & - & - \\
\hline
\end{tabular}

Obs.: No tratamento com chlorimuron foi utilizado o óleo mineral Assist a $0,5 \%$ (v/v) em pós-emergência.

${ }^{1} \mathrm{G} . \mathrm{D}=$ grânulos dispersíveis. $/{ }^{2} \mathrm{C} . \mathrm{E} .=$ concentrado emusionável.

${ }^{3}$ Dose: i.a. (ingrediente ativo), p.c. (produto comercial).

Tabela 2. Características dos produtos utilizados no experimento para o controle de Sida rhombifolia na cultura da soja. Embrapa Soja. Londrina-PR, 1997.

\begin{tabular}{|c|c|c|c|}
\hline Nome com um & Nome Químico & Grupo Químico & $\begin{array}{c}\text { Classe } \\
\text { Toxicológica }\end{array}$ \\
\hline Diclosulam & $\begin{array}{l}\mathrm{n} \text {-( }(2,6 \text {-dichloropheni1 })-5 \text { etoxy-7 fluoro }(1,2,4) \\
\text { triazolo- }(1,5 \text { c) pyrimidi-2-sulfonamide }\end{array}$ & triazolopirimidina & II \\
\hline Metolachlor & $\begin{array}{l}\text { 2-cloro-N-(2-etil-6-metifenil)-N-(2-metoxi-1- } \\
\text { metiletil) acetamida }\end{array}$ & acetanilidas & II \\
\hline Lactofen & $\begin{array}{l}1^{\prime}-(\text { carboetoxi)etil } 5-(2-\text { cloro-4-(trifluoro- } \\
\text { metil)fenoxi)-2-nitrobenzoato }\end{array}$ & difenil-éteres & III \\
\hline Chlorimuron-ethyl & $\begin{array}{l}\text { etil } 2-((((4-\text { cloro- } 6-\mathrm{m} \text { etoxi-pirimidina }-2- \\
\text { il)amina }) \text { carbonil }) \text { a m ino }) \text { sulfonil }) \text { benzoato }\end{array}$ & sulfoniluréias & I \\
\hline
\end{tabular}

\section{RESULTADOS E DISCUSSÃO}

Aos 35 dias após a aplicação dos tratamentos com a menor dose de diclosulam ( $25 \mathrm{~g} / \mathrm{ha})$, isolado ou em mistura com metolachlor, foram tão eficientes no controle da espécie estudada quanto os de maiores doses, não diferindo destes estatisticamente (Tabela 4). A variação na percentagem de controle esteve entre $93,7 \%$ com diclosulam a $25 \mathrm{~g} / \mathrm{ha}$ e $98,7 \%$ na mistura com metolachlor. Quando se utilizou metolachlor isoladamente, ficou em evidência o aumento de controle, proporcional ao da dose aplicada. Estes tratamentos não diferiram estatisticamente entre si, mas foram diferentes das misturas de metolachor com diclosulam ou deste produto isoladamente. Devido às condições climáticas adversas, a época de aplicação do tratamento padrão, chlorimurom + lactofen, foi atrasada, e acabou ocorrendo quando as plantas estavam fora do estádio previsto, que era de 3 a 4 folhas, o que pode justificar a baixa eficiência de controle (52,5\%). O chlorimuron necessita de um período mínimo de 15 dias para expressar seu efeito, o que não havia ocorrido nesta primeira avaliação.

Aos 60 dias os tratamentos com diclosulam isolado ou em mistura com metolachlor continuaram com elevados níveis de controle (acima de 95\%), e os tratamentos com metolachlor isoladamente continuaram, comparativamente, com baixa eficiência, assim como o tratamento padrão. As diferenças foram estatisticamente significativas.

Diferenças estatísticas continuaram a ocorrer também na avaliação aos 91 dias com a menor dose de metolachlor isoladamente $(1200 \mathrm{~g} / \mathrm{ha})$ que apresentou nível de controle inferior a todos os tratamentos com diclosulam. Porém, nas doses mais altas, as diferenças estatísticas desapareceram e somente os tratamentos com diclosulam foram equivalentes 
à testemunha capinada. Quanto ao tratamento padrão, observou-se aumento na porcentagem de controle devido ao desenvolvimento da cultura, cujo sombreamento ajudou a debilitar ainda mais as plantas daninhas já atingidas pelo produto químico. Embora muitas plantas não tenham morrido, o efeito supressor foi grande.

Tabela 3. Precipitações pluviais observadas antes e após a instalação do experimento (dezembro/96 e janeiro/ 97). Embrapa Soja. Londrina-PR, 1997.

\begin{tabular}{|c|c|c|}
\hline \multirow{2}{*}{ Dia } & \multicolumn{2}{|c|}{ Precipitação $(\mathrm{mm})$} \\
\hline & Dez-96 & Jan-97 \\
\hline 1 & 1,6 & 0,0 \\
\hline 2 & 10,0 & 0,0 \\
\hline 3 & 10,2 & 0,0 \\
\hline 4 & 16,1 & 0,0 \\
\hline $5^{*}$ & 3,2 & 0,0 \\
\hline $6 * *$ & 3,8 & 0,2 \\
\hline 7 & 13,2 & 0,0 \\
\hline 8 & 0,0 & 0,0 \\
\hline 9 & 0,0 & 12,0 \\
\hline 10 & 0,1 & 26,3 \\
\hline 11 & 14,7 & 7,3 \\
\hline 12 & 2,7 & 1,5 \\
\hline 13 & 0,0 & 0,0 \\
\hline 14 & 0,0 & 0,0 \\
\hline 15 & 0,0 & 1,4 \\
\hline 16 & 1,5 & 0,9 \\
\hline 17 & 28,5 & 0,5 \\
\hline 18 & 0,2 & 7,0 \\
\hline 19 & 0,0 & 26,3 \\
\hline 20 & 11,2 & 59,4 \\
\hline 21 & 0,4 & 36,1 \\
\hline 22 & 9,3 & 27,0 \\
\hline 23 & 0,0 & 3,9 \\
\hline 24 & 0,0 & 1,5 \\
\hline 25 & 12,1 & 5,0 \\
\hline 26 & 0,0 & 8,0 \\
\hline 27 & 0,0 & 6,3 \\
\hline 28 & 51,5 & 14,1 \\
\hline 29 & 40,0 & 5,2 \\
\hline 30 & 12,1 & 1,1 \\
\hline 31 & 0,0 & 21,7 \\
\hline Total & 242,4 & 272,7 \\
\hline
\end{tabular}

(*) Semeadura, (**) Aplicação tratamento padrão.
A aplicação dos métodos para a avaliação de controle, proposta pela Sociedade Brasileira da Ciência das Plantas Daninhas (SBCPD, 1995), indicou que todos os tratamentos com diclosulam isolado ou em mistura com metolachlor, apresentaram excelente controle similar à testemunha capinada. Os tratamentos com metolachlor isoladamente, foram descritos como moderados ou insuficientes na menor dose e como moderado a bom nas maiores doses. O tratamento padrão foi considerado, no máximo, como moderado comparativamente aos demais (Tabela 4).

O padrão, chlorimuron + lactofen, mostrou a maior percentagem de injúria à cultura $(12,5 \%$ aos 10 dias após a aplicação) estando no entanto, bem abaixo de 30\%, o limite a partir do qual o dano é considerado prejudicial, com possibilidade de interferência na produção (Tabela 5). Na avaliação realizada aos 60 dias os sintomas visuais reduziram ou desapareceram, conforme o tratamento. Os tratamentos atingiram no máximo a descrição de danos leves, com rápida recuperação, insuficientes para promover reduções de produtividade (SBCPD, 1995).

A variação na produtividade entre todos os tratamentos foi de 3397 a $2695 \mathrm{~kg} / \mathrm{ha}$, incluindo a testemunha capinada. Apesar da população da invasora ter sido em média de 69 plantas $/ \mathrm{m}^{2}$, a análise estatística mostrou diferenças apenas entre a testemunha sem capina e os tratamentos com diclosulam a $35 \mathrm{~g} / \mathrm{ha}$ e diclosulam a $25 \mathrm{~g} / \mathrm{ha}$ misturado com metolachlor a 1200 e $1800 \mathrm{~g} / \mathrm{ha}$. Embora tenha ocorrido seca no início de desenvolvimento da cultura, a ponto de atrasar a aplicação do tratamento padrão, o clima foi considerado chuvoso o suficiente para que o fator água não chegasse a ser limitante, principalmente no período de enchimento de grãos. A precipitação totalizou 242,3, 272,7 e 209,4 mm em dezembro, janeiro e fevereiro, respectivamente (Tabela 3). A altura das invasoras em nenhum momento superou a altura da cultura e o solo foi considerado de alta fertilidade. Estes fatores podem ajudar a explicar o baixo nível de dano ocorrido na testemunha capinada, expresso pela produção.

\section{CONCLUSÕES}

A mistura diclosulam + metolachlor, quando aplicada em pré-emergência, nas doses de $25+1200 \mathrm{~g} / \mathrm{ha}$, apresentou excelente nível de controle da Sida rhombifolia.

O diclosulam sozinho a 25 e $35 \mathrm{~g} / \mathrm{ha}$, apresentou excelente nível de controle da guanxuma.

O metolachlor nas maiores concentrações apresentou controle satisfatório da guanxuma.

Nenhum dos tratamentos apresentou fitotoxicidade que prejudicasse o desenvolvimento e a produtividade da cultura da soja. 
Tabela 4. Controle de Sida rhombifolia aos 35, 60 e 91 dias após a aplicação (DAA) de herbicidas em pré-emergência na cultura da soja. Embrapa Soja. Londrina-PR, 1997.

\begin{tabular}{|c|c|c|c|c|c|c|c|}
\hline \multirow{3}{*}{ Tratamento } & \multirow{3}{*}{$\begin{array}{c}\text { Dose } \\
\text { (g/ha) }\end{array}$} & \multicolumn{6}{|c|}{ Cotrole $(\%)$} \\
\hline & & \multicolumn{6}{|c|}{ Dias Após os Tratamentos } \\
\hline & & 35 & $\mathrm{CT}^{3}{ }^{3}$ & 60 & CT. & 91 & CT. \\
\hline Diclosulam & 25 & $93,7 \mathrm{a}^{/ 2}$ & $\mathrm{~A}$ & $96,2 a^{2}$ & A & $195,7 \mathrm{abc} /{ }^{2}$ & $\mathrm{~A}$ \\
\hline Diclosulam & 35 & $97,5 \mathrm{a}$ & A & $95,0 \mathrm{a}$ & A & $96,2 \mathrm{abc}$ & A \\
\hline Diclosulam + metolachlor & $25+1200$ & $98,7 \mathrm{a}$ & A & $99,5 \mathrm{a}$ & A & $99,5 \mathrm{a}$ & A \\
\hline Diclosulam + metolachlor & $25+1800$ & $97,5 \mathrm{a}$ & A & $98,2 \mathrm{a}$ & A & $99,0 \mathrm{a}$ & $\mathrm{A}$ \\
\hline Diclosulam + metolachlor & $35+1200$ & $97,5 \mathrm{a}$ & A & $97,7 \mathrm{a}$ & A & $97,5 \mathrm{ab}$ & A \\
\hline Diclosulam + metolachlor & $35+1800$ & $97,5 \mathrm{a}$ & A & 99,2 a & A & 99,0 a & $\mathrm{A}$ \\
\hline Metolachlor & 1200 & $70,0 \mathrm{~b}$ & $\mathrm{C}$ & $52,5 \mathrm{~cd}$ & $\mathrm{C}$ & $66,6 \mathrm{~d}$ & $\mathrm{C}$ \\
\hline Metolachlor & 1800 & $73,7 \mathrm{~b}$ & $\mathrm{C}$ & $68,7 \mathrm{bc}$ & $\mathrm{C}$ & $81,2 \mathrm{bcd}$ & $\mathrm{B}$ \\
\hline Metolachlor & 2400 & $80,0 \mathrm{~b}$ & $\mathrm{C}$ & $76,2 \mathrm{~b}$ & $\mathrm{C}$ & $82,5 \mathrm{bcd}$ & $\mathrm{B}$ \\
\hline Chlorimuron + lactofen $/ 1$ & $12,5+96$ & $52,5 \mathrm{c}^{1}$ & $\mathrm{D}$ & $50,0 \mathrm{~d} /{ }^{1}$ & $\mathrm{D}$ & $80,0 \mathrm{~cd} /{ }^{1}$ & $\mathrm{C}$ \\
\hline Testemunha s/capina & - & $0,0 \mathrm{~d}$ & E & $0,0 \mathrm{e}$ & E & $0,0 \mathrm{e}$ & $\mathrm{E}$ \\
\hline Testemunha c/capina & - & $100 \mathrm{a}$ & A & $100 \mathrm{a}$ & A & $100 \mathrm{a}$ & A \\
\hline $\mathrm{F}$ & & 79,89 & & 67,83 & & 110,09 & \\
\hline Coeficiente de variação $(\%)$ & & 5,85 & & 9,63 & & 6,33 & \\
\hline D.M.S $(5 \%)$ & & 5,17 & & 8,27 & & - & \\
\hline
\end{tabular}

${ }^{1}=$ Padrão (Pós-emergente). Leitura aos 10, 29 e 49 D.A.A.

${ }^{2}=$ Médias na coluna seguidas pela mesma letra não diferem entre si pelo teste de Tuckey a $5 \%$ de probabilidade. Dados analisados na forma original (não transformados).

${ }^{13}=$ Conceitos aplicados a avaliações de controle (SBCPD, 1995).

$\mathrm{A}=$ Controle excelente ou total; $\mathrm{B}=$ Controle bom ou aceitável; $\mathrm{C}=$ Controle moderado ou insuficiente; $\mathrm{D}=$ Controle deficiente ou inexpressivo;

$\mathrm{E}=$ Ausência de controle.

Tabela 5. Fitotoxicidade dos tratamentos na cultura da soja aos 35, 60 e 91 dias após a aplicação (DAA) e rendimento de grãos. Embrapa Soja. Londrina-PR, 1997.

\begin{tabular}{|c|c|c|c|c|c|c|}
\hline \multirow{3}{*}{ Tratamento } & \multirow{3}{*}{$\begin{array}{c}\text { Dose } \\
\text { (g/ha) }\end{array}$} & \multicolumn{4}{|c|}{ Fitotoxicidade (\%) } & \multirow{3}{*}{$\begin{array}{c}\text { Rendimento } \\
\text { da Soja } \\
(\mathrm{kg} / \mathrm{ha})\end{array}$} \\
\hline & & \multicolumn{4}{|c|}{ Dias Após a Semeadura } & \\
\hline & & 35 & $\mathrm{CT}^{3}{ }^{3}$ & 60 & CT. & \\
\hline 1. Diclosulan & 25 & $1,2 \mathrm{~b} /{ }^{2}$ & $\mathrm{~B}$ & $0,0 \mathrm{a} /{ }^{2}$ & $\mathrm{~A}$ & $3131 \mathrm{ab} / 2$ \\
\hline 2. Diclosulan & 35 & $2,5 \mathrm{~b}$ & B & $0,7 \mathrm{a}$ & $\mathrm{B}$ & 3394 a \\
\hline 3. Diclosulan + metolachlor & $25+1.200$ & $0,0 \mathrm{~b}$ & A & $0,0 \mathrm{a}$ & A & 3397 a \\
\hline 4. Diclosulan + metolachlor & $25+1.800$ & $5,0 \mathrm{~b}$ & B & $0,5 \mathrm{a}$ & B & 3389 a \\
\hline 5. Diclosulan + metolachlor & $35+1200$ & $0,0 \mathrm{~b}$ & A & $0,0 \mathrm{a}$ & A & $3301 \mathrm{ab}$ \\
\hline 6. Diclosulan + metolachlor & $35+1800$ & $5,0 \mathrm{~b}$ & $\mathrm{~B}$ & $1,2 \mathrm{a}$ & $\mathrm{B}$ & $2931 \mathrm{ab}$ \\
\hline 7. Metolachlor & 1200 & $0,0 \mathrm{~b}$ & A & $0,0 \mathrm{a}$ & A & $3110 \mathrm{ab}$ \\
\hline 8. Metolachlor & 1800 & $1,2 \mathrm{~b}$ & $\mathrm{~B}$ & $0,0 \mathrm{a}$ & A & $3218 \mathrm{ab}$ \\
\hline 9. Metolachlor & 2.400 & $1,2 \mathrm{~b}$ & $\mathrm{~B}$ & $0,0 \mathrm{a}$ & A & $3193 \mathrm{ab}$ \\
\hline 10. Chlorimuron + lactofen $/{ }^{1}$ & $12,5+96$ & $12,5 \mathrm{a} /{ }^{1}$ & B & $3,0 \mathrm{a} /{ }^{1}$ & B & $3046 \mathrm{ab}$ \\
\hline 11. Testemunha s/capina & - & $0,0 \mathrm{~b}$ & A & $0,0 \mathrm{a}$ & A & $2695 \mathrm{~b}$ \\
\hline 12. Testemunha c/capina & - & $0,0 \mathrm{~b}$ & A & $0,0 \mathrm{a}$ & A & $3218 \mathrm{ab}$ \\
\hline $\mathrm{F}$ & & 6,36 & & 1,97 & & 2,93 \\
\hline Coeficiente de variação \% & & 121,5 & & 230,6 & & 7,1 \\
\hline D.M.S. $(5 \%)$ & & 7,23 & & 3,22 & & - \\
\hline
\end{tabular}

$\mu^{1}=$ Padrão ( Pós-emergente). Leitura aos 10, 29 e 49 D.A.A.

${ }^{2}=$ Médias na coluna seguidas pela mesma letra não diferem entre si pelo teste de Tuckey a 5\% de Probabilidade. Dados analisados na forma original.

$\beta=$ Conceitos aplicados a avaliações de fitotoxicidade (SBCPD, 1995).

$\mathrm{A}=$ Sem injúria. Sem efeito sobre a cultura; $\mathrm{B}=$ Injúrias leves e/ou reduções de crescimento com rápida recuperação. Efeitos insuficientes para promover reduções de produtividade; $C=$ Injúrias moderadas e/ou reduções de crescimento com lenta recuperação ou definitivas. Efeitos intensos o suficiente para promover pequenas reduções de produtividade. $\mathrm{D}=$ Injúrias severas e/ou reduções de crescimento não recuperáveis e/ou reduções de estande. Efeitos intensos o suficiente para promover drásticas reduções de produtividade; E= Destruição completa da cultura ou somente algumas plantas vivas. 


\section{LITERATURA CITADA}

DEUBER, R. Ciência das plantas daninhas: fundamentos. Jaboticabal: FUNEP, 1992. 431p.

EMBRAPA SOJA. Recomendações técnicas para a cultura da soja no Estado do Paraná 1996/1997. Londrina: Embrapa Soja, 1996. 187p. (Embrapa Soja, Documentos, 97).
LORENZI, H. Plantas daninhas do Brasil: terrestres, aquáticas, parasitas, tóxicas e medicinais. Nova Odessa, SP: Editora Plantarum, 1982. 308p.

SOCIEDADE BRASILEIRA DA CIÊNCIA DAS PLANTAS DANINHAS. Procedimentos para instalação, avaliação e análise de experimentos com herbicidas. Londrina: SBCPD, 1995. 42p. 